\title{
How Do Patients Expect Apps to Provide Drug Information?
}

\author{
Anton Grube, Tobias Dehling, Ali Sunyaev \\ Information Systems and Systems Engineering \\ Research Center for IS Design (ITeG) \\ University of Kassel, Germany \\ \{grube, tdehling, sunyaev\}@ uni-kassel.de
}

\begin{abstract}
Patients use various sources to obtain information on pharmaceutical drugs. Mobile health care applications (apps) providing drug information to users are increasingly made available and of rising importance for the health care domain. However, apps usually offer functionality that only medical professionals or vendors consider useful for patients, although their considerations are not likely to meet patient expectations. In our exploratory study, we identify 33 features patients expect in apps for drug information provision with interviews and empirically assess their perceived importance in an online survey. Results indicate that patients desire personalization features for provided information but not for the app interface. This work contributes to research and practice by identifying and empirically ranking drug information provision features patients find important. We furthermore establish a foundation for future research on effective mobile drug information provision and provide insights for practice on development of patient-centered mobile health apps.
\end{abstract}

\section{Introduction}

Medical professionals should not ask what information their patients could provide to them but what information they should provide to their patients. Dating back to the Hippocratic Oath such behavior seems obvious, but today more important than ever. A brief look at the current health information systems (IS) landscape reveals strong affection for the big data hype. Large quantities of personal information are merged to pseudonymized profiles that represent average needs and demands of all patients and mask the needs and demands of the individual patient.

In contrast, the overall health care domain is undergoing a sweeping shift from paternalism to patient-centered health care [1]. In the past, health care was focused on generalization and the population level. Nowadays, patient-centered health care instead focuses on patients' individual needs [2]. Pharmaceutical drugs are used in health care to cure or ameliorate symptoms of an illness or a medical condition. Lacking drug adherence, contributing to higher treatment costs, poor health care outcomes, loss of confidence, and higher morbidity and mortality rates, is a core issue in health care $[3,4]$. Patients can intentionally refuse or unintentionally forget to take prescribed drugs. In the latter case, active reminders can familiarize patients with a prescribed regimen, make them accustomed to drug taking, and help to reduce non-adherence [5], whereas, the former case is often caused by a lack of information on drugs [6]. Provision of information on drugs patients take or intend to take is crucial for the success of therapy but seldom meets patients' needs and demands.

The rapid growth of mobile health information technology (IT) as well as the increasing mobile network expansion spurred the development of mobile health IS targeting patients. Ubiquitous information access, led to a wide spectrum of mobile health IS promoting patients' drug adherence through drug information provision features. For instance, online drug information databases, acoustic reminders, or text messages delivered through smartphone applications (apps) [7], clearly demonstrate the potential of current technology to provide information that patients demand [5]. However, medical professionals and vendors remain the key interest groups. Consequently, information creation and development of mobile health IS features for patients is often based on requirements, objectives, and expectations of medical professionals and vendors. Patients serve only as recipients of drug information. Little is known on what features patients expect in mobile health IS for drug information provision and how patients expect these features to help them to improve their drug adherence.

Extant research on evaluation and assessment of smartphone health apps for drug adherence is also often based on the expectations and demands of interest groups other than patients. Quality benchmarks for mobile health IS features (eg, usefulness for pa- 
tients) are usually not based on patients' but on researchers' or medical professionals' expectations [5, 8-10]. Hence, developers tend to tailor mobile health IS offerings to comply with expectations of researchers and medical professionals. However, neither medical professionals' nor researchers' expectations of features to be offered in mobile health IS targeting patients are likely to match patients' actual expectations, requirements, and demands. Consequently, provided drug information is often not understood, of no avail to patients, and fails to make an impact on patients' drug adherence.

To move forward, our research takes a step back and investigates the main interest group's (patients') expectations for features to be offered in mobile health IS focused on drug information provision to improve patients' drug adherence. As an exploratory study towards better understanding patients' expectations for drug provision features, this paper answers the following research questions from the patient perspective: What features do drug information provision apps need to offer? What is the relative importance of identified features and what aspects of drug adherence are influenced by these features?

We solicit a study sample of potential users of mobile health IS and determine their expectations for features to be included in mobile health IS concerned with drug information provision. As a first phase, we conducted single-person, semi-structured interviews with a group of twelve potential users of drug information provision apps. The answers were summarized, structured, and grouped. For the second phase, 208 study participants were recruited with Amazon Mechanical Turk (AMT) [11] to find out how potential users expect the features identified in the first phase to impact their state of health, determine the importance of each feature, and identify the drug adherence facilitators users perceive to be impacted by the respective feature. The drug adherence facilitators were derived from the dimensions of a taxonomy of health IT and drug adherence [12].

Our work contributes to the scientific knowledge base by identifying and empirically ranking features for mobile drug information provision health IS desired by patients. We furthermore establish a foundation for future research on effective mobile drug information provision and provide insights for practice on development of patient-centered mobile health IS.

\section{Related Research}

\subsection{Drug Information Provision for Patients}

In the IS domain, the term 'information' refers to data associated, structured, and formed to meaningful facts [13]. 'Information' is used to transfer knowledge to a recipient or a group of recipients. Communicating information to recipients turns out difficult because individuals' information demands and level of expertise vary [14]. Hence, transmitted information often cannot be understood or is misinterpreted by individual recipients. This results in differences in understanding of communicated information for different individuals. Such differences in understanding are particularly critical in the health care domain (eg, for the care provider-patient communication relationship). To enhance comprehensibility of information, the same information must be adapted to the demands and expectations of different recipients, in order to be meaningful to all possible recipients. Adaption can, for example, be based on education, qualification, or demographics. Personalization based on individuals' demands and needs is an effective method of adapting information [15].

The relevance of information provision in today's health care landscape increases with the rising number of health IT solutions focusing on information provision for patients [16, 17]. Especially usercentered health IS for patients (patient-centered health IS) focus on tailored information provision for patients and must be primarily guided by the demands and expectations of patients. Drug information serves as a crucial information source for patients. However, drug information is created by pharmaceutical companies. Patients cannot affect and are not involved in drug information creation or its provision process. Yet, they are the most important recipients of drug information. This makes provision of tailored drug information challenging.

In contrast to patients, other interest groups can exert influence on provided information and on the provision process itself to further their objectives. Care providers, for instance, may be interested in providing as little side effect information as required by law to lower patients' worries and uncertainties [18]. In contrast, pharmacists are interested in comprehensive drug information provision to reduce patients' inquiries about missing information [19]. Since patients usually do not have a medical education, albeit carrying the sole responsibility to comply with prescribed regimens, patients require well-crafted drug information provision. Current drug information provision is however inconsistent, intransparent, contradictory, and contains inappropriate wording and technical terms [20]. Expectations for features of different interest groups are contradictory, and more importance is often given to medical professionals' expectations and demands instead of the requirements of patients. Mobile health IS features for drug information provision are often only a byproduct of 
companies' existing health IS. However, patients' demands need to be considered in effective mobile health IS for patients [21].

\subsection{Patient-Centered Health IT}

Patient-centered health care is concerned with care provision consistent with the values, needs, and expectations of patients and is most beneficial when medical professionals involve patients in health care discussions and decisions [1, 22]. Patient-centered health care focuses primarily on the well-being of the individual patient instead of the financial wellbeing of the overall healthcare industry [23]. Patientcenteredness implies that actions contributing to health care and treatment processes can be initiated by patients and decisions are made with patient consultation $[24,25]$. Consequently, patients do not feel disregarded and have coequal decision-making authority [26]. Patient-centered health care promises a number of benefits for all involved interest groups [27-29]. When patients consult online health IS, they seek for additional information on their treatment (eg, in case of dissatisfaction with prescribed treatment plans) and expect to find reasonable advice helping to manage their own care [30, 31]. However, patients usually do not have sufficient medical expertise to assess the whole picture of their state of health and to estimate the consequences of their decisions [32]. Hence, medical professionals still must steer patients' decisions and anticipate wrong decisions through additional information provision [32, 33]. In the end, patients and medical professionals must together shoulder the greater responsibility that comes with the greater decision-making power of patients [34].

Patient involvement in treatment processes can proceed in different ways [29, 35]: Patients can provide additional relevant information about their health condition, self-quantification devices can be employed to gather vital signs during daily life activities, or patients can share experiences with a drug or a treatment [32]. Shared information strengthens the decision process for medical professionals [26]. Patients' experiences offer insights and reveal issues often not considered during conventional medical check-ups [36]. The enhanced information base eases longitudinal analyses of patients' state of health by tracking disease courses, symptoms, and recovery processes [25, 37]. This allows patients to recognize potential issues and to contact medical professionals in a timely fashion [27, 29]. Information exchange with patients enables medical professionals to get new insights into courses of treatment and to avoid unforeseen pitfalls with other patients subject to similar diseases or treatment methods [38].

Patient-centered health care is a radical change in the very traditional health care domain and has the potential to increase patient satisfaction, quality of care, and overall outcomes through patient empowerment [39], if patients understand the provided information and if supported by a supportive health IS landscape [25, 37]. Health IS, tailoring provided information to patients' demands, empower patients to comprehend provided information.

\subsection{Personalization of Drug Information Provision}

Patient-centered mobile health IS offerings bear the potential to provide patients with tailored information. Thus, they must not be guided by one interest groups' demands and expectations and, in particular, not only by medical professionals' demands and expectations. Mobile health IS allow to offer personalization features that enhance users' capabilities to personalize provided information without offering individual information for each patient. Drug information can be personalized by hiding undesired or irrelevant information, such as pregnancy-related information for male users, and emphasizing important information, such as pregnancy-related information for pregnant female users. In general, personalization of information in form of text may be based on various factors, such as explicit personal details or implicit social environmental profiling [15]. Personalization could be automated, based on manually entered preferences, or rely on general usage patterns. In all cases, mobile health IS must offer technical assistance for personalization because patients may not be familiar with the possibilities and features offered by technology. Patients' activities must be guided, preventing them to lessen their state of health. Instead of offering patients more degrees of freedom for personalization, it seems more feasible to offer a set of features that do the tailoring of information for patients and reduce their effort to personalize the desired information. This would facilitate provision of drug information, reduce the risk of harmful consequences, and does not require costly, manual intervention by medical professionals.

In this research, we assess the information provision features for patient-centered mobile health IS by consolidating patients' expectations for drug information provision features and identifying the expected impact on patients' state of health from the patient perspective. Involving patients in our research is important to determine patients' viewpoint on features to be offered by mobile health IS for drug in- 
formation provision. The obtained catalog of desired features can inform future research projects by eliciting patients' perspectives and enables prioritization of important or desired features.

\section{Method}

Our study is split into a qualitative interview survey and a quantitative online survey phase. To obtain an overview of patients' expectations for features to be offered by mobile drug information provision health IS, qualitative, semi-structured, single-person interviews were conducted. Semi-structured interviews are an effective approach for requirement elicitation [40]. The result of the first phase of the study is a consolidated list of users' desired features for mobile drug information provision health IS and serves as input for the second study phase. In the second phase, users rated the features from phase one according to their importance. Furthermore, users were asked to judge which drug adherence facilitators are impacted by the respective feature.

\subsection{Qualitative Survey Phase Design}

The interviewees for the initial user survey were aware of the domain and topic of the study. The interviews were conducted by one researcher following an interview guide. In the beginning, the interviewer introduced the domain of health care and explained the concept of mobile drug information provision health IS and how they are intended to influence drug adherence through drug information provision. After the introduction, demographics were collected (eg, gender, age). In the following a scenario, where interviewees were asked to imagine themselves in a situation searching for online drug information, was introduced. In essence, participants were asked exactly one main question: "What features can you imagine in a web application for drug information provision?". Interviewees were also asked to describe desired features for mobile drug information provision health IS. Finally, all mentioned desired features were clearly named and described, duplicates were removed, and the features were grouped according to emerging patterns. Interviews were coded independently by two researchers to avoid bias.

\subsection{Facilitation of Drug Adherence}

To find out how patients perceive the features to impact drug adherence in the quantitative survey, we derived six drug adherence facilitators from a taxon- omy of health IT and medication adherence ${ }^{1}$ [12]. The dimensions of the taxonomy indicate which drug adherence facilitators are potentially enhanced through health IS features. Study participants were asked for a binary decision whether in their opinion the drug adherence facilitators are affected by the respective feature.

The first drug adherence facilitator is 'Seamless data input'. Ease of data input is crucial for users' future behavior. If users are obliged to perform many complex steps to import or enter personal health information in a mobile health IS, it is unlikely that they will use the feature. Easy and intuitive to use health IS features lead to increased usage, or usage intention, which in turn enables mobile health IS features to influence users' drug adherence. The second facilitator is 'Location- and device-independent access', which in general refers to the ability to make use of a mobile health IS feature independent of location or technical equipment. If users have the ability to make use of mobile health IS features on any device, mobile health IS use will increase and drug adherence may improve. The third facilitator is 'Collaboration with medical professionals or other users'. Collaboration between users and medical professionals or with other users is referred to: working jointly towards a better state of health or better health care provision. An ongoing process of sharing information with each other and the possibility to benefit for both sides from personal experiences or frequently occurring problems leads to enhanced drug literacy and hence to reduced error rate when taking drugs. The fourth drug adherence facilitator is 'Quality control by medical professionals'. Most health care tasks involving mobile health IS can be executed by users without the assistance of medical professionals. However, some tasks require qualified input from medical professionals or other experts (eg, to assess blood values). Medical professionals have the highest authority in health care and are trained to provide reliable health-related information. Involving medical professionals in the care process, enhances information provision through reliable information on drugs (eg, if users do not know how to behave in case of unexpected side effects). The fifth facilitator is 'Obtaining health-related feedback'. Feedback on health-related topics, in form of motivational messages, adjustment of a treatment plan, or treatment progress notifications, is supported by many mobile health IS offerings and gives users insight into their personal state of health. User-focused feedback on

\footnotetext{
${ }^{1}$ The original taxonomy of health IT and medication adherence identified seven dimensions (drug adherence facilitators). However, for this study six facilitators were used since 'information storage' was not consistently interpreted by study participants.
} 
health-related issues relieves medical professionals from common requests. The sixth drug adherence facilitator is 'Personalization'. Personalization of mobile health IS is, for example, often realized through the adjustment of font size, hiding unwanted content (eg, text sections or functions), or reorganizing content. Personalized mobile health IS offer tailored information provision to users and reduce users' effort for understanding the provided information by displaying only relevant information (eg, hiding dosage for children for childless adults).

\subsection{Quantitative Survey Phase}

The online survey questionnaire started with a description of the domain and a brief instruction for the participants. Participants were asked to rate features on five point Likert scales according to their perceived importance (1='not important', 5='very important') and to select one or more drug adherence facilitators that may be positively impacted by the respective feature. Before finishing the survey, participants were asked for demographics and given the opportunity to make suggestions for additional features or drug adherence facilitators not addressed in the study.

The survey was carried out in three batches at different times via the labor market Amazon Mechanical Turk (AMT) with one day in between each batch to avoid daytime, or worker characteristic biases. To avoid common method biases the items were formulated in simple language, unfamiliar terms were defined, examples were provided where necessary, and double-barreled questions were decomposed [41]. On the first and the second day, 50 survey participants were solicited. Survey results were reviewed after each batch. The advantage of AMT, compared with conventional survey designs, where participants must be acquired on a voluntary basis, motivated with gifts, vouchers, or payments, is that AMT offers easy access to a high number of potential participants. AMT is a common tool in behavioral research and study sample, sample quality, and sample domain can be tailored to the needs and requirements of a particular study [42]. The number of participants can flexibly be set and is usually reached in a very short period of time (1 hour for 50 participants). Extant research demonstrates that survey results with AMT participants have high reliability, and provide higher quality data than student or public online surveys [11, 43-45]. Participants choose the tasks they want to perform. Payment depends on the complexity and duration of the task and can be freely defined. In total we paid each study participant US\$1.20 for the completion of the survey. We paid participants US\$1.0 for completing the survey. Furthermore, we paid an additional US $\$ 0.20$ because we solicited only experienced participants who had previously completed at least 5,000 tasks with an approval rate of $99 \%$ to ensure high data quality [46].

\section{Results}

\subsection{Qualitative Survey Phase}

Overall twelve participants were interviewed ( $6=$ male, $6=$ female $)$ with an average age of 35 years (min. age $=20$, max. age=50). In total, 90 desired features were elicited. After removal of duplicates and features irrelevant for drug information provision (eg, finding medical professionals, price search engine for drugs), 33 features remained, and were classified in four groups: 'Information provision features' (INFO), 'Graphical user interface features' (GUI), 'Search and sort features' (SEARCH), and 'Features providing additional functionality’ (FUNC) (cf. Table 1).

\subsection{Quantitative Survey Phase}

Overall 208 U.S. participants responded to the survey. Each worker could only participate once in the survey. 18 participants (9\%) aborted the survey, 20 $(10 \%)$ were excluded because of wrong control answers, too short survey response time (under 3 minutes for a 10-minute survey), and suspicious answer schemes (eg, same value for all features). 168 (81\%) participants completed the online survey ( $89=$ male, $79=$ female) with age between 20 and 64 years. $60(36 \%)$ participants were younger than 30 years, $53(32 \%)$ participants were between 30 and 39 years old, $36(21 \%)$ participants were between 40 and 49 years old, 17 (10\%) participants were between 50 and 59 years old, and 2 (1\%) participants were over 60 years old. $87(52 \%)$ participants had a university degree, $55(33 \%)$ participants had a vocational- (24; $14 \%)$, a secondary- $(17 ; 10 \%)$, or a general- $(14 ; 8 \%)$ school degree, $9(5 \%)$ had an international Baccalaureate, $8(5 \%)$ finished school with no qualification, 6 (4\%) were still in school, and 1 MBA-, 1 Associate's, and 1 J.D. degree. Most of the participants (103; $61 \%$ ) were employed, 40 (24\%) were self-employed, $11(7 \%)$ were unemployed, $7(4 \%)$ were university students, $2(1 \%)$ civil servants, $2(1 \%)$ retired, and 3 (2\%) participants were on disability insurance.

Overall participants gave positive response values on importance (mean across all features $=3.80$, min. mean $=2.79$, max. mean $=4.6,95 \%$ confidence interval=0.06). Table 1 lists the elicited features from the quantitative study phase, ordered by mean importance and perceived associations with drug 
Table 1. Desired features for drug information provision in health IS.

\begin{tabular}{|c|c|c|c|c|c|c|c|c|c|c|}
\hline \multirow{3}{*}{\multicolumn{2}{|c|}{ / Featu }} & \multirow{2}{*}{\multicolumn{3}{|c|}{ Importance }} & \multirow{2}{*}{\multicolumn{6}{|c|}{$\begin{array}{l}\text { Perceived associations with drug } \\
\text { adherence facilitators: } \mathbf{n}(\%)\end{array}$}} \\
\hline & & & & & & & & & & \\
\hline & & $\sum^{\Xi}$ & 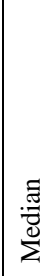 & 穴 & 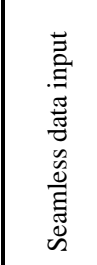 & 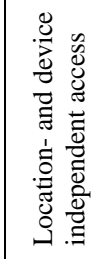 & 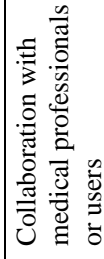 & 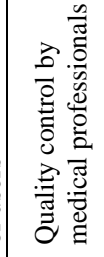 & 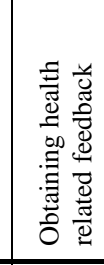 & 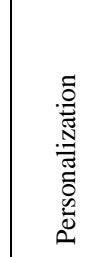 \\
\hline 1 & FUNC: Check drugs for adverse drug reactions. & 4.60 & 5.0 & 0.75 & $15(05)$ & $13(05)$ & $61(22)$ & $94(33)$ & $62(22)$ & $38(13)$ \\
\hline 2 & $\begin{array}{l}\text { GUI: Well-arranged presentation of drug information (eg, clear } \\
\text { emphasis of headings, good structure and readability). }\end{array}$ & 4.58 & 5.0 & 0.68 & $59(22)$ & $17(06)$ & $48(18)$ & $63(24)$ & $49(19)$ & $28(11)$ \\
\hline 3 & SEARCH: Search drugs by name. & 4.42 & 5.0 & 0.86 & $96(36)$ & $19(07)$ & $35(13)$ & $42(16)$ & $34(13)$ & $41(15)$ \\
\hline 4 & $\begin{array}{l}\text { GUI: Display dosage information based on other factors than age } \\
\text { (eg, weight, height, vital values). }\end{array}$ & 4.17 & 4.5 & 1.00 & $23(08)$ & $8(03)$ & $57(20)$ & $84(29)$ & $36(13)$ & $77(27)$ \\
\hline 5 & GUI: Simple user interface design. & 4.09 & 4.0 & 0.88 & $98(32)$ & $37(12)$ & $39(13)$ & $36(12)$ & $41(13)$ & $59(19)$ \\
\hline 6 & INFO: Provide information whether a drug unit can be split. & 4.08 & 4.0 & 0.99 & $16(06)$ & $17(07)$ & $51(21)$ & $79(32)$ & $39(16)$ & $45(18)$ \\
\hline 7 & $\begin{array}{l}\text { GUI: Display dosage information for a drug in different units (for } \\
\text { example: } \mathrm{ml}, \mathrm{mg}, \mathrm{g}, \mathrm{cl} \text {, pills). }\end{array}$ & 4.06 & 4.0 & 1.04 & $30(11)$ & $10(04)$ & $51(19)$ & $88(33)$ & $29(11)$ & $55(21)$ \\
\hline 8 & FUNC: Compare two or more drugs (eg, side-effects). & 4.06 & 4.0 & 0.92 & $31(12)$ & $12(05)$ & $54(22)$ & $66(26)$ & $44(18)$ & $43(17)$ \\
\hline 9 & SEARCH: Search drugs by application area/medical condition. & 4.04 & 4.0 & 0.94 & $63(25)$ & $19(08)$ & $49(19)$ & $52(21)$ & $34(13)$ & $36(14)$ \\
\hline 10 & $\begin{array}{l}\text { INFO: Provide information on drugs which may be taken to } \\
\text { alleviate side effects. }\end{array}$ & 4.03 & 4.0 & 0.97 & $16(06)$ & $10(04)$ & $63(23)$ & $71(26)$ & $66(24)$ & $48(18)$ \\
\hline 11 & $\begin{array}{l}\text { INFO: Provide information on how to enhance the effect of a } \\
\text { taken drug (eg, through diet or specific behavior). }\end{array}$ & 3.98 & 4.0 & 0.91 & $13(05)$ & $13(05)$ & $47(20)$ & $55(23)$ & $54(23)$ & $57(24)$ \\
\hline 12 & SEARCH: Filter and search for side-effects (eg, by occurrence). & 3.98 & 4.0 & 1.01 & $47(16)$ & $17(06)$ & $51(17)$ & $58(20)$ & $50(17)$ & $72(24)$ \\
\hline 13 & INFO: Provide information if a drug can be dissolved in a fluid. & 3.98 & 4.0 & 1.02 & $14(06)$ & $10(04)$ & $49(21)$ & $71(31)$ & $39(17)$ & $49(21)$ \\
\hline 14 & $\begin{array}{l}\text { SEARCH: Filter search results by users' personal characteristics } \\
\text { (eg, based on age, allergies, or personal preferences). }\end{array}$ & 3.96 & 4.0 & 1.09 & $57(19)$ & $13(04)$ & $48(16)$ & $45(15)$ & $44(14)$ & $101(33)$ \\
\hline 15 & FUNC: Provide the possibility to store drugs users are taking. & 3.92 & 4.0 & 1.16 & $55(19)$ & $18(06)$ & $43(15)$ & $43(15)$ & $42(15)$ & $86(30)$ \\
\hline 16 & FUNC: Provide a tutorial on how to use the application. & 3.91 & 4.0 & 1.03 & $47(23)$ & $17(08)$ & $31(15)$ & $32(16)$ & $23(11)$ & $52(26)$ \\
\hline 17 & INFO: Provide information how a drug acts in your body. & 3.90 & 4.0 & 0.97 & $12(05)$ & $12(05)$ & $41(18)$ & $54(23)$ & $65(28)$ & $48(21)$ \\
\hline 18 & $\begin{array}{l}\text { FUNC: Provide the ability to store users' personal information } \\
\text { (eg, age, weight, allergies). }\end{array}$ & 3.87 & 4.0 & 1.12 & $71(23)$ & $14(04)$ & $37(12)$ & $41(13)$ & $41(13)$ & $111(35)$ \\
\hline 19 & SEARCH: Search drugs by active ingredient. & 3.80 & 4.0 & 1.05 & $59(25)$ & $19(08)$ & $41(18)$ & $53(23)$ & $28(12)$ & $32(14)$ \\
\hline 20 & FUNC: Allow users to comment on their experiences with a drug. & 3.80 & 4.0 & 1.08 & $31(10)$ & $10(03)$ & $75(24)$ & $33(11)$ & $75(24)$ & $83(27)$ \\
\hline 21 & \begin{tabular}{|l|} 
INFO: Provide additional information for the technical terms or \\
abbreviations (eg, links to explanations, dictionaries, synonyms).
\end{tabular} & 3.76 & 4.0 & 1.12 & $23(11)$ & $12(06)$ & $45(21)$ & $63(29)$ & $45(21)$ & $27(13)$ \\
\hline 22 & FUNC: Provide functionality to identify drugs (eg, shape, color). & 3.75 & 4.0 & 1.07 & $38(19)$ & $10(05)$ & $33(16)$ & $61(30)$ & $33(16)$ & $29(14)$ \\
\hline 23 & $\begin{array}{l}\text { INFO: Provide information on follow-up drugs (eg, after the } \\
\text { maximum application period of a drug exceeded). }\end{array}$ & 3.73 & 4.0 & 1.00 & $15(06)$ & $9(04)$ & $66(28)$ & $65(27)$ & $52(22)$ & $32(13)$ \\
\hline 24 & FUNC: Provide functionality to print out selected information. & 3.67 & 4.0 & 1.12 & $36(18)$ & $21(11)$ & $26(13)$ & $37(19)$ & $31(16)$ & $49(25)$ \\
\hline 25 & $\begin{array}{l}\text { FUNC: List all other drugs with same active ingredient as cur- } \\
\text { rently displayed drugs (eg, generic drugs). }\end{array}$ & 3.64 & 4.0 & 1.08 & $21(09)$ & $17(07)$ & $62(27)$ & $66(29)$ & $31(14)$ & $31(14)$ \\
\hline 26 & $\begin{array}{l}\text { SEARCH: Search drugs by a unique local identifier (eg, NDC, } \\
\text { PZN, UDI). }\end{array}$ & 3.57 & 4.0 & 1.24 & $83(33)$ & $22(09)$ & $41(16)$ & $44(17)$ & $30(12)$ & $33(13)$ \\
\hline 27 & SEARCH: Filter for requirement of prescription. & 3.55 & 4.0 & 1.09 & $53(21)$ & $13(05)$ & $53(21)$ & $47(19)$ & $31(12)$ & $57(22)$ \\
\hline 28 & $\begin{array}{l}\text { FUNC: List all other drugs for the same application area/medical } \\
\text { condition as currently displayed drugs. }\end{array}$ & 3.40 & 3.5 & 1.12 & $22(10)$ & $11(05)$ & $61(29)$ & $59(28)$ & $32(15)$ & $26(12)$ \\
\hline 29 & GUI: Integrate images/pictograms to illustrate drug information. & 3.24 & 3.0 & 1.12 & $30(16)$ & $14(08)$ & $24(13)$ & $34(18)$ & $32(17)$ & $50(27)$ \\
\hline 30 & SEARCH: Search drugs by company/manufacturer. & 3.23 & 3.0 & 1.22 & $65(30)$ & $20(09)$ & $35(16)$ & $46(21)$ & $22(10)$ & $28(13)$ \\
\hline 31 & FUNC: Provide image of drug package. & 3.06 & 3.0 & 1.18 & $22(14)$ & $13(08)$ & $19(12)$ & $54(34)$ & $17(11)$ & $34(21)$ \\
\hline 32 & INFO: Provide information on homespun remedies. & 2.86 & 3.0 & 1.35 & $10(06)$ & $10(06)$ & $33(19)$ & $27(16)$ & $37(21)$ & $56(32)$ \\
\hline 33 & GUI: Customizable user interface. & 2.79 & 3.0 & 1.25 & $33(13)$ & $25(10)$ & $25(10)$ & $14(05)$ & $21(08)$ & $144(55)$ \\
\hline & Average and total values: & 3.80 & 3.9 & 1.94 & $\begin{array}{l}1304 \\
(16 \%)\end{array}$ & $\begin{array}{l}502 \\
(06 \%)\end{array}$ & $\begin{array}{l}1494 \\
(18 \%)\end{array}$ & $\begin{array}{l}1777 \\
(22 \%)\end{array}$ & $\begin{array}{l}1309 \\
(16 \%)\end{array}$ & $\begin{array}{l}1757 \\
(22 \%)\end{array}$ \\
\hline
\end{tabular}


adherence facilitators. Based on these results, the derived features were classified into three groups: 'Essential features', 'Nice-to-have features', and 'Convenience features' (cf. Figure 1). Essential features (4 items) were rated most important for drug information provision mobile health IS. Nice-to-have features (23 items) represent the class of contextspecific features, that may be irrelevant for some user groups but advantageous for other users (eg, a tutorial on app usage may be superfluous by experienced users but desired for unexperienced users). Convenience features ( 6 items) are features with the lowest importance ratings, these features may offer useful functionality for some users of mobile drug adherence health IS. Figure 1 shows the breakdown by average feature rating in groups.

Most features facilitate drug adherence through 'Quality control by medical professionals' and 'Personalization' (both 22\%). At the end of the study, participants were asked to comment on the study or to propose features, which were not elicited in the first phase of our study. Most participants $(130 ; 77 \%)$ ignored the free text field or noted, that the amount of features leaves nothing more to expect. However, 38 participants responded with detailed feedback on the study and detailed feature descriptions for mobile drug provision health IS. A noticeable amount of feedback is concerned with demand for a feature establishing communication between care-providers or pharmacists and patients. For instance, survey participants stated:

"I did feel the option to see other users' feedback/opinions would be incredibly valuable."

"Ability to communicate with a doctor"

"I might add some kind of functionality to allow the user to get in contact with a pharmacist."

We selectively address further constructive answers in the discussion section. A complete list of participants' feedback as well as the complete survey results is available from the authors upon request.

\section{Discussion}

This study investigates features desired by users for drug information provision through mobile health IS. It is apparent from the qualitative survey phase that users are mostly interested in features enabling them to refine provided drug information and to quickly find certain information (eg, drug information on alternative or similar drugs for the same application area). Demand for personalization or individualization of the provided drug information is lower. Refinement and personalization features can both enable patients to tailor provided drug infor- mation to their needs and demands. Previous research discovered that provided drug information usually exceeds the amount of information required by patients [20]. Hence, patients demand features that enable them to hide unwanted or irrelevant information or to enrich the provided information with explanations or additional information [47].

Results of the quantitative survey phase demonstrate that features for adverse drug reaction check, search features, and well-arranged drug information presentation are the most desired features. This is also supported by extant research in the domain of clinical pharmacology [48]. Features like search drugs by manufacturer (\#30), show image of a drug package (\#31), show information on homespun remedies (\#32), and customization of the graphical user interface (\#33) are the least desired features. However, these features also received high importance ratings by several survey participants (29 (17\%), 21 (13\%), 25 (15\%), and 19 (11\%) participants gave the highest possible rating, respectively). These results indicate that users may underestimate the riskiness of features. Features \#32 and \#33 may, for example, bear risks by providing dangerous information (eg, ineffective homespun remedies [49]) or through accidental hiding of relevant information on drugs through wrong personalization preferences.

When it comes to seeking health information online, most users are motivated by an illness or a problematic situation [50]. Users search for information helping them to make the right decisions to solve the problem. Uncontrollable problems, in particular uncontrollable health problems attributed to wrong drug application, may cause serious harm to users' state of health [4, 51]. Hence, users want to receive the desired information, and to take action quickly. Our survey supports these findings by a low demand for the feature: "Customizable user interface." (\#33, mean=2.79) (cf. Table 1). Users expect easy-to-use user interfaces without or with little setup effort, which for instance, two participants explicitly requested:

"Other than making it easy to find info on medications, no.”

"Just make it as easy to use as possible. [...] they [people] can be easily confused."

Furthermore, survey participants mentioned that they prefer information whether drugs are covered by users' health insurance. For instance, one survey participant stated:

„Also would it be possible (or impossible!) to load insurance information such as co-pays, deductibles, coinsurance, etc. by insurance provid- 


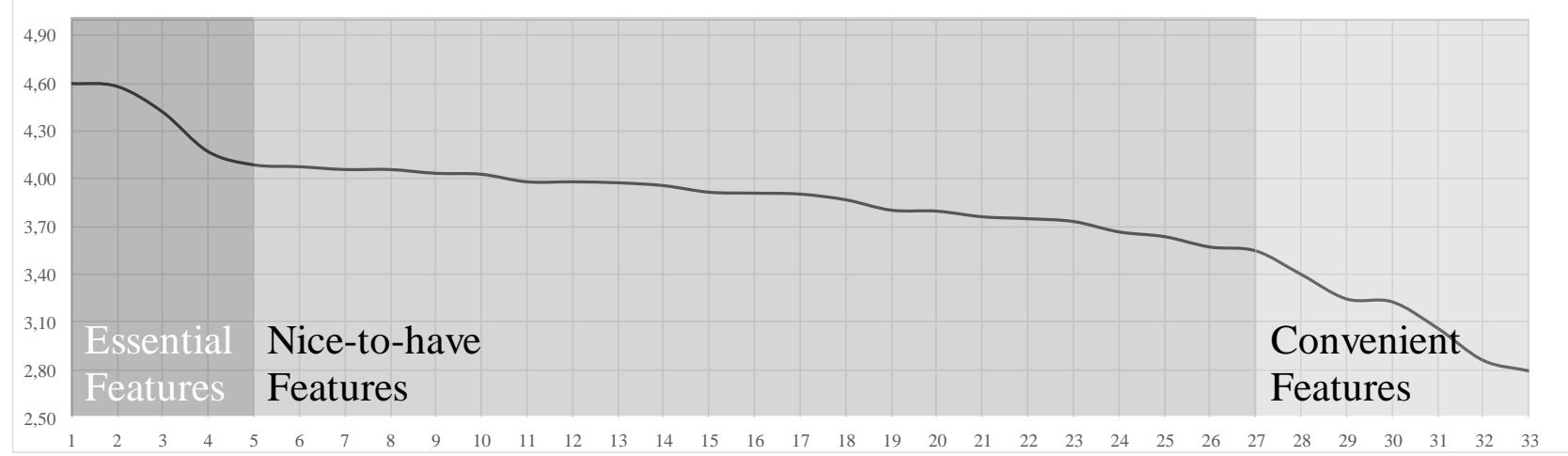

Figure 1. Mean importance values for all features. (Vert.-axis: Mean importance; Hor.-axis: Feature \#)

er, so that a US patient would be able to know if they could afford to take the medicine or not? "

The quantitative survey phase reveals associated drug adherence facilitators for each feature but also gives a general overview of most often associated facilitators over all features. Overall, the most influential drug adherence facilitators for the analyzed features are: 'Quality control by medical professionals' (22\%), 'Personalization' (22\%), and 'Collaboration with medical professionals or users" (18\%) (cf. Table 1). Extant research supports our findings on usefulness of social support for patients to find relevant information in social networks [52]. Since features fostering communication between medical professionals or other users were not included in our survey, many survey participants explicitly mentioned to consider these features (cf. Section 4.2).

In contrast to the qualitative phase, where user interface personalization features were frequently mentioned in personal interviews, our findings from the quantitative phase reveal the exact opposite. Users expect personalization features only for the provided drug information, which would result in lower effort to obtain the demanded information. These findings are supported by high ratings for personalization features (\#4, mean=4.17; \#14, mean=3.96) and by participants' responses in the open text answer section of the survey. For instance, two participants stated:

"A personal journal section where users can write down their experiences [...] can be helpful to keep track of how effective certain treatments on a personal basis. The patient could easily track their thoughts, benefits, and side-effects of each medication they are taking in one place online."

"[...] Most [features] were geared towards personalization."

Many features, frequently mentioned in the quantitative survey and highly demanded by patients in the quantitative study are not offered in today's mo- bile health IS landscape. In the qualitative survey phase, participants often mentioned features, that allow to check for adverse drug reactions or to compare drugs (eg, by side-effects). In the quantitative phase, importance of these features for patients was confirmed through high ratings $(\# 1$, mean $=4.6$; \#8, mean $=4.06$ ). These results indicate, that patients need information on drug compatibility and on negative consequences when taking specific drugs. In contrast, mobile health IS offerings available today seem rather focus on covering many platforms to offer their features to patients (eg, availability of apps offering features to improve drug adherence on multiple mobile OS platforms) or the implementation of fancy technologies (eg, cloud computing features) fostering location and device independent access to information. In our study, the drug adherence facilitator: "Location- and device independent access" is least often associated with features for drug information provision, which indicates patients' unawareness or lack of interest for features enabling patients to store personal drug information in cloud services or accessing them with different devices. Furthermore, extant research results reveal a demand for mobile health IS offering a wide range of features and enhanced levels of functionality [9]. Our study results reveal that patients find only a small set of features very important. Additional features could be added to target specific patient groups and diversify offered mobile health IS. Offering functionality for a designated interest group or domain could meet expectations more effectively, reduces sources for errors, and offers greater benefit for users. This would also entail easier system design due to a smaller set of features to develop, to test, and to maintain and purposeful attention to specific needs of a specific group of users.

Our study provides insights on the relative importance of drug information provision features for users but does not provide insights on other interest groups in health care. Hence, future research should 
investigate whether and how the identified features are important to medical professionals, pharmacists, and vendors. Given that desired features are highly dependent on factors such as experiences or disease patterns, evaluating expected features under different contextual conditions or comparing expectations of different health care interest groups seem to be promising research directions. Furthermore, it is unclear whether features demanded by patients, can be beneficial for patients and improve patients drug adherence. After careful elaboration and design, the efficacy of these features must be tested and established in real world settings (eg, with a clinical trial).

Limitations of our study affect the following aspects. First, although our sample size for the qualitative phase (12 respondents) is sufficient to provide an estimate of patients desired features, it is relatively small. A larger sample size may include further user groups and identify further desired features. Second, the quantitative study is limited to one country. Hence, a larger sample might incorporate perspectives from different countries with different health care expectations. Third, the quantitative survey was carried out via the labor market AMT with a participants group restricted to the U.S. Different sampling strategies may lead to different results. Furthermore, inclusion of international participants from other regions than the U.S., might also lead to different feature ratings and overall different survey outcomes. A larger sample size would also allow to account for differences in financial status and insurance situation. A promising opportunity would be to explore users' expectations for features for drug information provision across cultures. Fourth, our research only considers users' expectations for features for drug information provision. We did not cover features that users' do not want in drug information provision apps, which might reveal different insights. Furthermore, features appearing important in the qualitative survey phase, turned out not important in the quantitative study phase (eg, interface personalization), which indicates that patients' demands may vary due to various reasons. Features may seem important on paper but are perceived as unimportant when implemented.

The results of our research contribute to the development of patient-centered mobile health IS. Identifying patients' demands and preferences for mobile health IS features and implementing these in 'readyto-use' mobile health IS offerings promotes patient involvement, participation and empowerment. Design and development of mobile health IS targeting drug adherence in form of mobile apps based on patients' ideas and beliefs might lead to more frequent use. This may in turn lead to higher effectiveness of these apps and improve health care outcomes.

\section{References}

[1] Mead, N. and Bower, P., "Patient-Centredness: A Conceptual Framework and Review of the Empirical Literature", Soc Sci Med, 51(7), 2000, pp. 1087-1110.

[2] Stewart, M., "Towards a Global Definition of Patient Centred Care: The Patient Should be the Judge of Patient Centred Care", Brit Med J, 322(7284), 2001, pp. 444-445.

[3] Naderi, S.H., Bestwick, J.P., and Wald, D.S., "Adherence to Drugs that Prevent Cardiovascular Disease: MetaAnalysis on 376,162 Patients", Am J Med, 125(9), 2012, 882-887.e1.

[4] Osterberg, L. and Blaschke, T., "Adherence to Medication", New Engl J Med, 353(5), 2005, pp. 487-497.

[5] Stawarz, K., Cox, A.L., and Blandford, A., "Don't Forget Your Pill!", in Proceedings ACM Conference on $\mathrm{Hu}-$ man Factors in Computing Systems, 2014, ACM, New York, NY, USA.

[6] Sabaté, E., "Adherence to Long-Term Therapies: Evidence for Action", World Health Organization Library Cataloguing-in-Publication Data, Zürich, 2003.

[7] Sunyaev, A., et al., "Availability and Quality of Mobile Health App Privacy Policies", J Am Med Inform Assn, 22(e1), 2015, e28-e33.

[8] Sunyaev, A., et al., "Evaluation Framework for Personal Health Records: Microsoft HealthVault Vs. Google Health", in Proceedings 43th Hawaii International Conference on System Sciences, 2010, IEEE.

[9] Dayer, L., et al., "Smartphone Medication Adherence Apps: Potential Benefits to Patients and Providers", J Am Pharm Assoc, 53(2), 2013, pp. 172-181.

[10] Heldenbrand, S., et al., "Assessment of Medication Adherence App Features, Functionality, and Health Literacy Level and the Creation of a Searchable Web-Based Adherence App Resource for Health Care Professionals and Patients", J Am Pharm Assoc, 56(3), 2016, pp. 293-302.

[11] Buhrmester, M., Kwang, T., and Gosling, S.D., "Amazon's Mechanical Turk: A New Source of Inexpensive, Yet High-Quality, Data?", Perspect Psychol Sci, 6(1), 2011, pp. 3-5.

[12] Mrosek, R., Dehling, T., and Sunyaev, A., "Taxonomy of Health IT and Medication Adherence", Health Policy and Technology, 4(3), 2015, pp. 215-224.

[13] Checkland, P. and Holwell, S., Information, Systems and Information Systems: Making Sense of the Field, John Wiley \& Sons, Chichester, New York, Weinheim, 1998.

[14] Paris, C.L., "Tailoring Object Descriptions to a User's Level of Expertise", Comput Linguist, 14(3), 1988, pp. 6478.

[15] Kaptein, M., et al., "Personalizing Persuasive Technologies: Explicit and Implicit Personalization Using Persuasion Profiles", Int J Hum-Comput St, 77, 2015, pp. 3851.

[16] Forster, A., et al., "Information Provision for Stroke Patients and their Caregivers", Cochrane Db Syst Rev, 1996.

[17] Willis, A.M., et al., "How do Prospective Parents Prefer to Receive Information about Prenatal Screening and 
Diagnostic Testing?", Prenatal Diag, 35(1), 2015, pp. 100102 .

[18] Bassetti, S., et al., "Why is Highly Active Antiretroviral Therapy (HAART) not Prescribed or Discontinued?", J Acq Imn Def, 21(2), 1999, pp. 114-119.

[19] Holappa, M., et al., "Information Sources Used by Parents to Learn About Medications they are Giving their Children", Res Social Adm Pharm, 8(6), 2012, pp. 579584.

[20] Mossman, J., "How Much Information is too Much for Patients?", J Risk Res, 2015, pp. 1-4.

[21] Klecun, E., "Transforming Healthcare: Policy Discourses of IT and Patient-Centred Care", Eur J Inf Syst, 25(1), 2015, pp. 64-76.

[22] Dehling, T. and Sunyaev, A., "Secure Provision of Patient-Centered Health Information Technology Services in Public Networks", Electronic Markets, 24(2), 2014, pp. 89-99.

[23] Kleinke, J.D., "Dot-Gov: Market Failure and the Creation of a National Health Information Technology System", Health Aff, 24(5), 2005, pp. 1246-1262.

[24] Eysenbach, G., "Medicine 2.0: Social Networking, Collaboration, Participation, Apomediation, and Openness", J Med Internet Res, 10(3), 2008, e22.

[25] Swan, M., "Health 2050: The Realization of Personalized Medicine Through Crowdsourcing, the Quantified Self, and the Participatory Biocitizen", J Pers Med, 2(3), 2012, pp. 93-118.

[26] Shaw, J. and Baker, M., "“Expert Patient" - Dream or Nightmare?", Brit Med J, 328(7442), 2004, pp. 723-724.

[27] Eysenbach, G., "Consumer Health Informatics", Brit Med J, 320(7251), 2000, pp. 1713-1716.

[28] Kahn, J.S., Aulakh, V., and Bosworth, A., "What it Takes: Characteristics of the Ideal Personal Health Record", Health Aff, 28(2), 2009, pp. 369-376.

[29] Ricciardi, L., et al., "A National Action Plan to Support Consumer Engagement via E-Health", Health Aff, 32(2), 2013, pp. 376-384.

[30] Li, N., et al., "Reasons for and Predictors of Patients' Online Health Information Seeking Following a Medical Appointment", Fam Pract, 31(5), 2014, pp. 550-556.

[31] Wilkins, A.S., "Expanding Internet Access for Health Care Consumers", Health Care Manage R, 24(3), 1999, pp. 30-41.

[32] Hamilton, D.W., "Shared Decision Making Asks Patients to Share their Aims and Values for Treatment", Brit Med J, 348, 2014, g1435.

[33] Davies, P., "Should Patients be Able to Control Their Own Records?", Brit Med J, 345, 2012, e4905.

[34] Anderson, R.M. and Funnell, M.M., "Patient Empowerment: Myths and Misconceptions", Patient Educ Couns, 79(3), 2010, pp. 277-282.

[35] Coulter, A. and Ellins, J., "Effectiveness of Strategies for Informing Educating and Involving Patients", Brit Med J, 335(7609), 2007, pp. 24-27.
[36] Horwitz, R.I., et al., "(De)Personalized Medicine", Science, 339(6124), 2013, pp. 1155-1156.

[37] Parish, E., "BMJ Roundtable Debate: How Can we get Better at Providing Patient Centred Care?", Brit Med J, 350, 2015, h412.

[38] Stacey, D., et al., "Decision Aids for People Facing Health Treatment or Screening Decisions", Cochrane Db Syst Rev(1), 2014, CD001431.

[39] Richards, T., Coulter, A., and Wicks, P., "Time to Deliver Patient Centred Care", Brit Med J, 350, 2015, h530.

[40] Davis, A., et al., "Effectiveness of Requirements Elicitation Techniques: Empirical Results Derived from a Systematic Review", in IEEE International Conference on Requirements Engineering, 2006, Minneapolis, MN, USA.

[41] Tourangeau, R., Rips, L.J., and Rasinski, K.A., The Psychology of Survey Response, Cambridge University Press, Cambridge, U.K., New York, 2000.

[42] Huang, E., et al., "Toward Automatic Task Design: A Progress Report", in Proceedings ACM SIGKDD Workshop, Washington DC, July 25 - 28, 2010.

[43] Behrend, T.S., et al., "The Viability of Crowdsourcing for Survey Research", Behav Res Methods, 43(3), 2011, pp. 800-813.

[44] Mason, W. and Suri, S., "Conducting Behavioral Research on Amazon's Mechanical Turk", Behav Res Methods, 44(1), 2011, pp. 1-23.

[45] Paolacci, G., Chandler, J., and Ipeirotis, P.G., "Running Experiments on Amazon Mechanical Turk", Judgm Decis Mak, 5(5), 2010, pp. 411-419.

[46] Peer, E., Vosgerau, J., and Acquisti, A., "Reputation as a Sufficient Condition for Data Quality on Amazon Mechanical Turk", Behav Res Methods, 46(4), 2014, pp. 1023-1031.

[47] Zeng-Treitler, Q., Kim, H., and Hunter, M., "Improving Patient Comprehension and Recall of Discharge Instructions by Supplementing Free Texts with Pictographs", AMIA Annu Symp Proc, 2008, pp. 849-853.

[48] Dooren, A.A., et al., "Communication Between Patients and Pharmacy Staff on Patient Information Leaflets", J Adv Clin Pharmacol, 2(2), 2015, pp. 39-49.

[49] Thomas, S.H. and Stone, C., "Acute Toxicity from Baking Soda Ingestion", Am J Emerg Med, 12(1), 1994, pp. 57-59.

[50] Cline, R. J. W. and K.M. Haynes, "Consumer Health Information Seeking on the Internet: The State of the Art", Health Educ Res, 16(6), 2001, pp. 671-692.

[51] Ho, P.M., Bryson, C.L., and Rumsfeld, J.S., "Medication Adherence: Its Importance in Cardiovascular Outcomes", Circulation, 119(23), 2009, pp. 3028-3035.

[52] Nambisan, P., "Information Seeking and Social Support in Online Health Communities: Impact on Patients' Perceived Empathy", J Am Med Inform Assn, 18(3), 2011, pp. 298-304. 